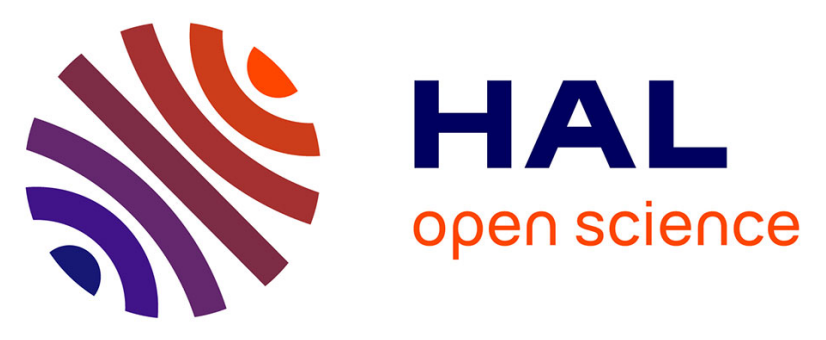

\title{
Condition of excitation and sensitivity of diffractively-coupled surface lattice resonances over plasmonic nanoparticle arrays in ATR geometry
}

Artem Danilov, Gleb Tselikov, Fan Wu, Vasyl G. Kravets, Igor Ozerov, Frédéric Bedu, Alexander Grigorenko, Andrei V Kabashin

\section{To cite this version:}

Artem Danilov, Gleb Tselikov, Fan Wu, Vasyl G. Kravets, Igor Ozerov, et al.. Condition of excitation and sensitivity of diffractively-coupled surface lattice resonances over plasmonic nanoparticle arrays in ATR geometry. Synthesis and Photonics of Nanoscale Materials XV, Jan 2018, San Francisco, United States. pp.1052109, 10.1117/12.2295192 . hal-01719970

\section{HAL Id: hal-01719970 \\ https://hal.science/hal-01719970}

Submitted on 13 Apr 2018

HAL is a multi-disciplinary open access archive for the deposit and dissemination of scientific research documents, whether they are published or not. The documents may come from teaching and research institutions in France or abroad, or from public or private research centers.
L'archive ouverte pluridisciplinaire HAL, est destinée au dépôt et à la diffusion de documents scientifiques de niveau recherche, publiés ou non, émanant des établissements d'enseignement et de recherche français ou étrangers, des laboratoires publics ou privés. 


\section{Condition of excitation and sensitivity of diffractively-coupled surface lattice resonances over plasmonic nanoparticle arrays in ATR geometry}

Artem Danilov, Gleb Tselikov, Fan Wu, Vasyl G. Kravets, Igor Ozerov, et al.

Artem Danilov, Gleb Tselikov, Fan Wu, Vasyl G. Kravets, Igor Ozerov, Frederic Bedu, Alexander N. Grigorenko, Andrei V. Kabashin, "Condition of excitation and sensitivity of diffractively-coupled surface lattice resonances over plasmonic nanoparticle arrays in ATR geometry ," Proc. SPIE 10521, Synthesis and Photonics of Nanoscale Materials XV, 1052109 (23 February 2018); doi: 10.1117/12.2295192 


\title{
Conditions of excitation and sensitivity of diffractively-coupled surface lattice resonances over plasmonic nanoparticle arrays in ATR geometry
}

\author{
Artem Danilov ${ }^{\mathrm{a}}$, Gleb Tselikov ${ }^{\mathrm{a}}$, Fan Wu $\mathrm{u}^{\mathrm{b}}$, Vasyl G. Kravets ${ }^{\mathrm{b}}$, Igor Ozerov ${ }^{\mathrm{c}}$, Frederic Bedu ${ }^{\mathrm{c}}$, \\ Alexander N. Grigorenko ${ }^{b}$, Andrei V. Kabashin ${ }^{a}$ \\ ${ }^{a}$ Aix Marseille Univ, CNRS, LP3, Marseille, France, 13288; \\ ${ }^{\mathrm{b}}$ School of Physics and Astronomy, University of Manchester, Manchester, UK, M13 9PL; \\ ${ }^{\mathrm{c}}$ Aix Marseille Univ, CNRS, CINAM, Marseille, France, 13288
}

\begin{abstract}
We investigate conditions of excitation and properties of Plasmonic Surface Lattice Resonances (PSLR) over glass substrate-supported Au nanoparticle dimers $(\sim 100-200 \mathrm{~nm})$ arranged in a periodic metamaterial lattice, in Attenuated Total Reflection (ATR) optical excitation geometry, and assess their sensitivities to variations of refractive index (RI) of the adjacent sample dielectric medium. We show that spectral sensitivity of PSLR to RI variations is determined by the lattice periodicity $(\sim 320 \mathrm{~nm}$ per RIU change in our case), while ultranarrow resonance lineshapes (down to a few nm full-widthat-half-maximum) provide very high figure-of-merit values evidencing the possibility of ultrasensitive biosensing measurements. Combining advantages of nanoscale architectures, including a strong concentration of electric field, the possibility of manipulation at the nanoscale etc, and high phase and spectral sensitivities, PSLRs promise a drastic advancement of current state-of-the-art plasmonic biosensing technology.
\end{abstract}

Keywords: plasmonic metamaterials for biosensing plasmonic surface lattice resonances, diffractive coupling, attenuated total reflection, plasmonic biosensing, improvement of sensitivity.

\section{INTRODUCTION}

Relying on control of biomolecular binding events by refractive index (RI) monitoring, plasmonic biosensing forms the core of current label-free biosensing technology, ${ }^{1,2}$. Conventional plasmonic biosensor employs Surface Plasmon Resonance (SPR) phenomenon, which is produced with a help of $50-\mathrm{nm}$ gold film in Kretschmann-Raether prism geometry ${ }^{1,2}$. Biomolecular interactions on gold lead to an increase of the thickness of a biomaterial layer, which is accompanied by a change of RI of the medium contacting the sensor surface. This results in a change of SPR coupling conditions, which can be monitored by angular ${ }^{1,2}$, spectral ${ }^{3}$ or phase ${ }^{4-6}$ interrogations. With the lower detection limit of 1 $\mathrm{pg} / \mathrm{mm}^{2}$ of biomaterial accumulated on the biosensor surface, SPR biosensors are efficient for studies of many biomolecular interactions ${ }^{7,8}$, but they are hardly compatible with current trends in biosensing focusing on the development of compact ${ }^{9-12}$ or nanoscale ${ }^{13-16}$ transducer implementations. Although localized plasmon resonances (LPR) over nanostructures ${ }^{13,14}$ look much better adapted for these modern trends of biosensing technology, they provide much higher detection limit of $\sim 1000 \mathrm{pg} / \mathrm{mm}^{2}$.

A huge potential for the upgrade of plasmonic biosensing technology is now expected from "plasmonic metamaterials for biosensing". These metamaterials were defined ${ }^{15}$ as artificial nanostructured materials, which can provide a much improved response compared to natural plasmonic materials $(\mathrm{Au}, \mathrm{Au})$ and/or enable new functionalities (size-related selectivity, concentration of electric field etc). Such a concept was implemented in numerous sensing designs, including plasmonic nanorod metamaterial ${ }^{15}$, hyperbolic metamaterial ${ }^{16}, 3 \mathrm{D}$ plasmon crystal metamaterial ${ }^{17}$. Of particular interest, we see the development of metamaterials, which enable the excitation of Plasmonic Surface Lattice Resonances (PSLR) produced in conditions of diffraction coupling of $\mathrm{LPR}^{18-22}$. To excite PSLR, gold nanoparticles (nanodics, nanopillars etc.) having the size of about $100 \mathrm{~nm}$ are arranged in a 2D lattice in such a way that one of diffracted beams, appearing due to

Synthesis and Photonics of Nanoscale Materials XV, edited by Jan J. Dubowski, Andrei V. Kabashin,

Linyou Cao, David B. Geohegan, Proc. of SPIE Vol. 10521, 1052109 - (c) 2018 SPIE

CCC code: $0277-786 \mathrm{X} / 18 / \$ 18 \cdot$ doi: $10.1117 / 12.2295192$ 
the periodicity of this structure, propagates over the array plane and can couple in far field localized plasmons over individual nanoparticles (Fig. 1a). Such a coupling leads to a drastic narrowing of resonances in reflected and transmitted light down to 2-3 nm full width at half maximum (FWHM). In addition, the diffraction coupling gives rise to vanishing of light intensity in resonances, leading to the generation of singularities of light phase $\mathrm{e}^{21,22}$. When used as a signal parameter to monitor refractive index variations ${ }^{23-25}$, such phase singularities can be used to lower the detection limit of label-free plasmonic biosensing schemes down to single molecule level ${ }^{21}$. However, conditions of implementation of these resonances are not always compatible with biosensing arrangement implying the placement of the nanoparticles between a glass substrate and a sample medium (air, water). In particular, the excitation of diffractively coupled PSLR is critically dependent on refractive index of the media surrounding a nanoparticle array. When the array is illuminated under normal incidence of light, the excitation of PSLR typically requires uniform surrounding, i.e. the match of refractive index of the substrate and that of the medium contacting the particles ${ }^{19,20}$. On the other hand, the excitation of PSLR becomes possible under oblique incidence of light on the array structure and the monitoring of optical parameters in reflected light ${ }^{18,21,22}$. In all cases, direct geometry of PSLR excitation is not fully compatible with biosensing experimental arrangement, as it implies light direction through a sample liquid leading to a dependence of PSLR parameters on bulk RI fluctuations inside the flow cell.

We recently showed ${ }^{26}$ that the excitation of PSLR is possible in both direct and attenuated total reflection (ATR) geometries under oblique light incidence. In this paper, we provide extensive details on conditions of excitation and properties of PSLR in ATR geometry, excluding the propagation of pumping light through sample liquid medium.

\section{EXPERIMENTAL METHODS}

\subsection{Sample preparation}

High-quality regular and homogenous square arrays of gold nanoparticles were produced by e-beam lithography (LEORAITH and PIONEER-RAITH) on a clean microscopic glass substrate covered by a thin Cr sublayer. A double layered resist was used to improve lift-off ( $80 \mathrm{~nm}$ of $495 \mathrm{kD}$ PMMA cast from a $3 \mathrm{wt} \%$ solution in anisole for the bottom resist layer and $50 \mathrm{~nm}$ of $95 \mathrm{kD}$ PMMA cast from a $2 \mathrm{wt} \%$ solution in anisole for the top layer). Bare (without $\mathrm{Cr}$ seed layer) borosilicate glass substrates (WBO-251 from UQG Optics) successively coated by a single layer of PMMA (from Allresist, Germany) diluted in ethyl-lactate at 2\%) is used for nanofabrication. Second layer of a conductive polymer (SX AR PC 5000/90.2 from Allresist) is applied in order to prevent the charging of the dielectric substrates during e-beam exposure. After developing in 1:3 MIBK:isopropanol solution, rinsing the samples in deionized water and drying under clean nitrogen flow, 3-5 $\mathrm{nm}$ of $\mathrm{Cr}$ (to improve adhesion) was deposited and 80-90 nm of Au by evaporation using electron beam or Joule effect (Auto 306 tool from Edwards). Then the excessive metal deposited onto the areas protected by the resist was removed from samples by the lift-off procedure in ultrasonic bath of pure ethyl-lactate. The typical array size was $0.2 \times 0.2 \mathrm{~mm}^{2}$. The samples on a clean glass substrate were obtained from the samples fabricated on a $5 \mathrm{~nm} \mathrm{Cr}$ sublayer in which the $\mathrm{Cr}$ sublayer has been wet-etched after the fabrication procedure. Typical Scanning Electron Microscopy images of double nanodot arrays are shown in Fig. 1.

\subsection{Methodology of measurements}

Measurements were performed on ellipsometer (Fig. 1c) in ATR configuration (Fig. 1d). The bottom side of the sample was mounted to the ground slope of a glass regular prism via optical immersion oil, this matched RI of glass. A gap for liquid flow was provided with rubber O-ring, fixed between the sample from one side and a fixed cover glass from the other. Weakly focused light beam of the source refracted on one slope of the prism, crossed prism insides, passed through the tiny layer of immersion oil and the sample substrate cover slip and then finally, reflected from the nanoarray. Incident and reflection beam paths were adjusted to be symmetrical with respect to the prism orientation. Device analyzed intensities of differently polarized light and phase shift between them. Reflection spectra dependency from angle of illumination were recorded. Data repeatability showed negligible variation of peak spectral position for each analyzed mixture, compared to system spectral resolution. 


\subsection{Sensitivity assessment}

In these experiments, the sensitivity of plasmonic metamaterial transducers was evaluated by using a model, which simulated changes of bulk refractive index of the aqueous medium contacting nanoparticle arrays, similarly to how it was done in previous studies ${ }^{23,24}$. A set of ethanol-water mixtures of different concentrations were prepared for evaluation of sample sensing capabilities. Ethanol has excellent solubility in water and does not affect the quality of glass-supported nanoparticle arrays. Since ethanol has slightly higher refractive index compared to water (1.3614 compared to 1.333 for $589 \mathrm{~nm}$ wavelength at room temperature), its progressive addition to aqueous solutions provides nearly linear increase of refractive index of the liquid. The mixtures were pumped though the liquid flow cell, as shown in Fig. 1c.
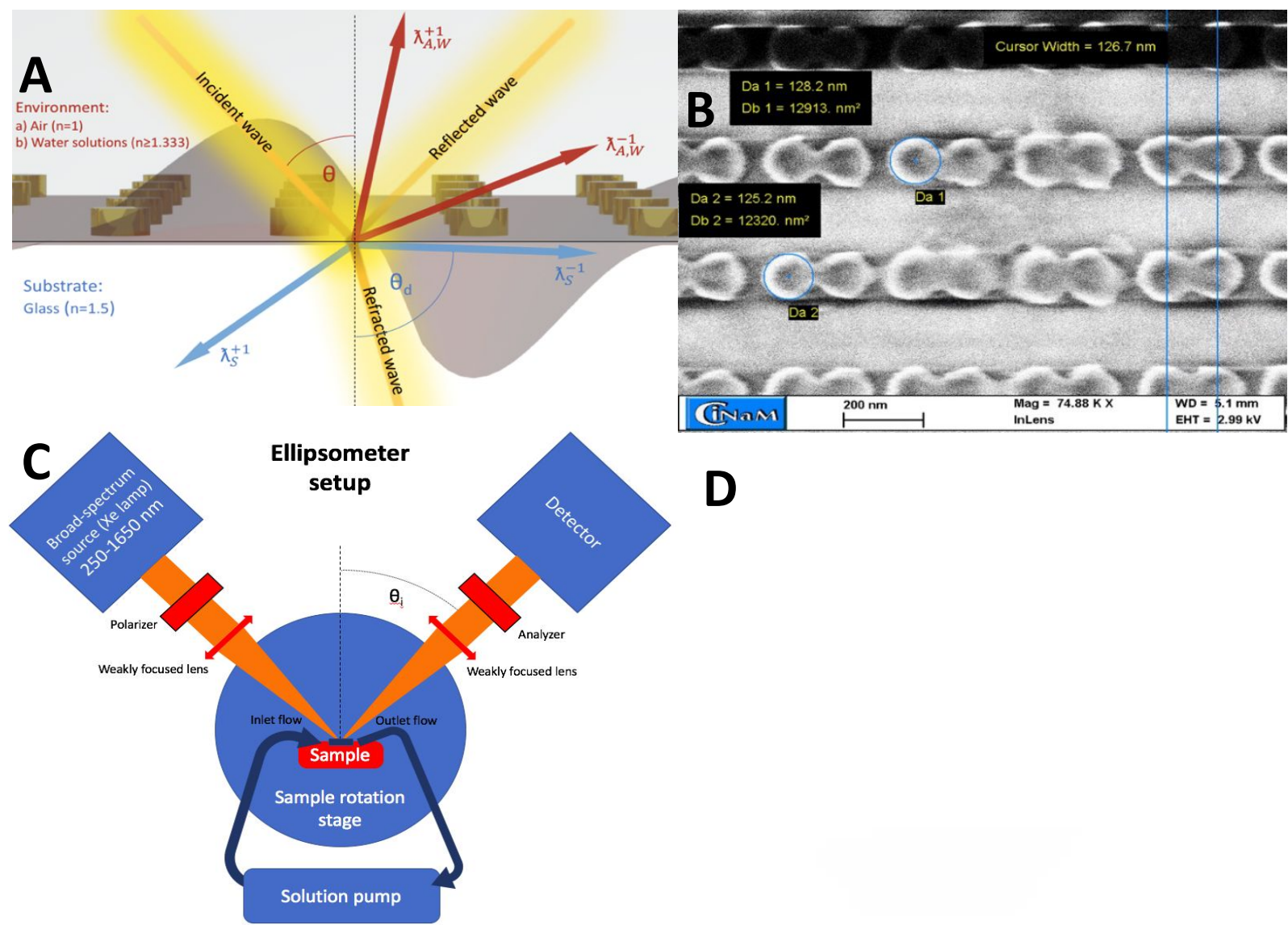

Figure 1. (a) Schematics of excitation of diffractively coupled plasmonic surface lattice resonances over a metal nanoparticle array. When metal nanoparticles are arranged in a periodic lattice, they may scatter light to produce diffracted waves. If one of the diffracted waves then propagates in the plane of the array, it may couple localized plasmon resonances associated with individual nanoparticles leading to the generation of PSLR with a very narrow lineshape. In the presence of a substrate, this phenomenon takes place at Rayleigh cut-off frequencies for the medium $\left(\lambda_{R}^{a i r}\right.$ or $\left.\lambda_{R}^{\text {wat }}\right)$ and substrate $\left(\lambda_{R}^{\text {sub }}\right)$; (b) Typical Scanning Electron Microscopy images of double nanoparticle arrays used for the excitation of PSLRs; (c) Schematic representation of ellipsometerbased experimental setup (d) Architecture of measurements using ATR geometry.

\section{RESULTS AND DISCUSSION}

\subsection{Conditions of excitation and properties of PSLR in ATR geometry}

First, we identify medium (PSLR air or simply A+, PSLR $_{\text {wat }}$ or W+ for air and water, respectively) and substrate (PSLR sub or $\mathrm{S}+$ ) modes corresponding to the coupling of individual plasmon oscillations at medium- and substrate-related diffraction cut-off edges. PSLR can be efficiently excited even under asymmetry of optical environment, but they split into two modes corresponding to the coupling of localized plasmons via diffracted waves propagating in the tested medium or the substrate (Fig. 1a). The reason of this splitting is the following: for any regular nanoparticle array, diffracted beam disappears when 
it crosses the boundary between ambient medium (air or water) and the substrate due to the impossibility of transition between media having essentially different light dispersion. As a result, the diffracted beam is cut-off at Rayleigh cut-off wavelength (diffraction edge). In the case when a medium/substrate interface is present, there should be two cut-off wavelengths. One of them corresponds to the disappearance of the medium diffraction modes (when the diffraction wave crosses the sample boundary from air/water to the substrate) and other one is related to crossing the boundary in the opposite direction. Such two Rayleigh cut-off wavelengths for air/substrate interface can be presented as follows ${ }^{18,26}$ :

$$
\lambda_{R}^{\text {air }}=a / m\left(n_{\text {air }} \pm \sin \theta\right), \lambda_{R}^{\text {sub }}=a / m\left(n_{\text {sub }} \pm \sin \theta\right)
$$

where $a$ is a period of the structure, $m$ is integer, $\theta$ is the angle of incidence, $n_{\text {sub }}$ is substrate RI. Similarly, for water/substrate interface Rayleigh cut-off wavelengths will be:

$$
\lambda_{R}^{w a t}=a / m\left(n_{w a t} \pm \sin \theta\right), \lambda_{R}^{\text {sub }}=a / m\left(n_{\text {sub }} \pm \sin \theta\right)
$$
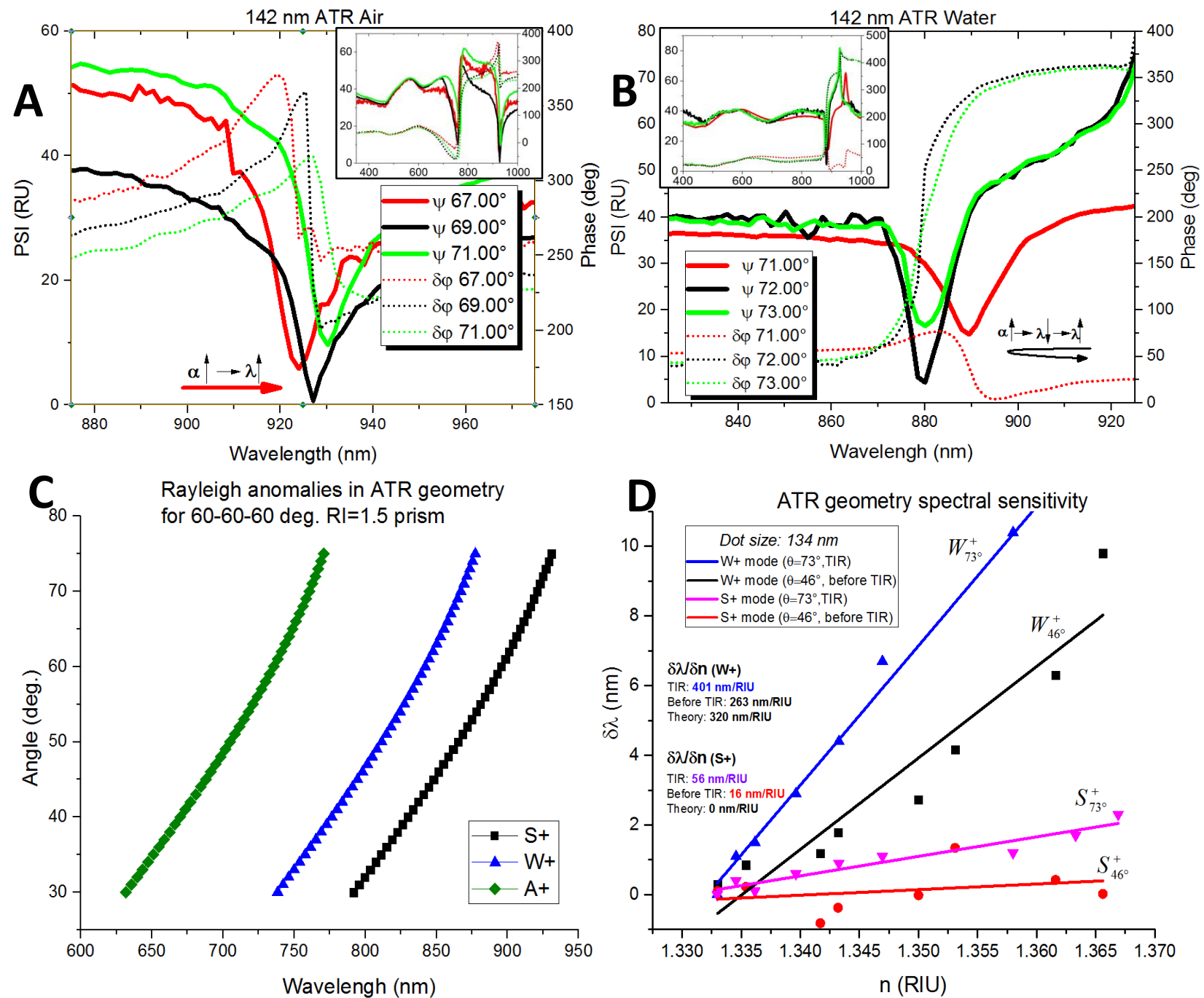

Figure 2. Spectral dependencies of ellipsometric reflectivity $\Psi$ (solid) and phase $\Delta$ (dashed) for a 320-nm period arrays of glasssupported gold nanoparticles contacting with air (a) and water (b) media under different angles of their illumination in ATR geometry. The insets show obtained spectra at minimized scale. (c) Analytically calculated spectral position of Rayleigh cutoff with respect to angle of incidence for water- $(\mathrm{W}+)$, air- $(\mathrm{A}+)$ and substrate-related $(\mathrm{S}+)$ modes. (d) Spectral response of medium PSLR wat $_{(\mathrm{W}+)}$ and PSLR $_{\text {sub }}(\mathrm{S}+)$ modes excited over double $134 \mathrm{~nm}$ particle array (period $320 \mathrm{~nm}$ ) before and after TIR to changes of RI. 
The effect of splitting of resonances can be easily seen in experiments. Fig. 2 presents spectral dependencies of ellipsometric parameters $\Psi$ and $\Delta$ for light reflected from a $320-\mathrm{nm}$ period golden double dot metamaterial arrays with 134-nm distance between the dot positions, having $\sim 50 \mathrm{~nm}$ separation, contacting with air and water media, respectively. As can be seen on the insets of Fig. 2, the resonances are indeed split into distinct medium- and substrate-related modes indicated as $\mathrm{A}+/ \mathrm{W}+$ and $\mathrm{S}+$, respectively, and they correlate with Rayleigh cut-off wavelengths $\lambda_{R}^{a i r} / \lambda_{R}^{w a t}$ and $\lambda_{R}^{\text {sub }}$. Fig. 2c shows spectral position of Rayleigh Anomalies (RA) for each media (air $\mathrm{A}_{+}^{+}$, water $\mathrm{W}+$ and substrate $\mathrm{S}+$ ) with respect to the angle of light incidence, calculated from (1) and (2). One can clearly see the correlation between calculated RA spectral position and the experimental data from double nanoparticle array. For instance, the inset of Fig. 2a shows two peaks with spectral positions roughly $750 \mathrm{~nm}$ and $930 \mathrm{~nm}$ at $\sim 70 \mathrm{deg}$. of light incidence. Fig. 2c shows, that these peaks correspond to calculated air and substrate Rayleigh cut-off frequencies, respectively. Similarly, $880 \mathrm{~nm}$ peak at $\sim 72 \mathrm{deg}$. of light incidence on Fig. 2b corresponds to water-related RA on Fig. 2c. PSLR typically appear in reflection as narrow Fano-shape dips over a positive background spectrum ${ }^{26}$, presenting the combination of reflected and scattered light from glass-supported nanoparticle array system. However, when measured by ellipsometry and presented as a function of ellipsometric reflection $\Psi$, which describes a ratio of amplitudes of $\mathrm{p}$ - and s- components upon reflection $\tan \Psi=\frac{\left|R_{p}\right|}{\left|R_{s}\right|}$, the resonances can appear as both minima (inset to Fig. 2a, left and right peaks; Fig. 2b, left peak) and maxima (inset to Fig. $2 \mathrm{~b}$, right peak). It is obvious that negative or positive polarity of $\operatorname{PSLR}_{\mathrm{s}}$ in $\Psi(\lambda)$ spectrum is determined whether resonant dips take place for p- or s-polarized component of light, respectively, or whether the substrate reflection is added to or subtracted from the reflection produced by the array. In general, the width and shape of PSLR critically depend on a variety of factors, including nanoparticle size, geometry of the unit cell and refractive index of the environment. In addition, the emergence of different modes and their intensity strongly depend on the angle of light incidence. Here, different structures appear to provide optimal resonance characteristics under different conditions. As an example, water-related mode $\mathrm{W}+$ (inset to Fig. 2b, left peak) is much more pronounced than water-related mode A+ (inset to Fig. 2a, left peak), reaching almost zero reflection intensity. Substrate-related modes are expected to show similar results no matter which media is applied to nanoparticle array. Surprisingly, the substrate-related peak shows polarization-dependent absorbance for the cases of nanoarray being exposed in air and water media. This effect is manifested as minima for case of air (Fig. $2 \mathrm{a}$, right peak) and maxima for water (Fig. 2b, right peak). The width of PSLR is also strongly dependent on array parameters and range from a few nm FWHM to 20-25 nm FWHM. In all cases, the implementation of ultra-narrow and high quality resonances requires the optimization of array structures and their adaptation for concrete biosensing architectures and environments (air, water). It is woth noting that the generation of these resonances is accompanied by a strong enhancement of local electric field ${ }^{27}$, which is very important for enabling additional sensing channels such as SERS.

\subsection{Sensitivity of PSLRs to local environment}

Ultimate biosensing experiment implies the functionalization of the plasmonic nanoparticles and immobilization of a sensor recognition element on them. A target analyte (affinity partner) comes from aqueous ambience and is supposed to bind to the recognition element leading to an increase of refractive index of a thin layer around the nanoparticles, while a relevant change of conditions of PSLR excitation (spectral, phase) enables one to monitor the RI increase caused by the biological interaction. To simulate the increase of RI of water ambience, aqueous solutions of ethanol of different concentrations were pumped through a flow cell by a peristaltic pump and brought into contact with the nanoparticle arrays.

Figure $2 \mathrm{~d}$ shows spectral shifts of resonances due to changes of refractive index of the aqueous medium caused by the addition of different concentration of ethanol to water. The refractive index (RI) of the medium increases with the ethanol addition leading to a nearly linear red shift of PSLR. However, sensitivities of medium and substrate-related modes appear to be quite different. Here, the value of sensitivity of the medium mode starts to depend whether its excitation takes place below or above the angle of total internal reflection (TIR) for glass/water interface $\left(\sim 63^{\circ} \mathrm{Deg}\right.$.). As follows from Fig. $2 \mathrm{~d}$, sensitivity of PSLR wat $(\mathrm{W}+)$ below the TIR angle $\left(\sim 46^{\circ} \mathrm{Deg}\right.$.) is also of the order of the period of the structure $(263 \mathrm{~nm} / \mathrm{RIU}$ for double $134 \mathrm{~nm}$ particle arrays), while for angles of incidence above TIR $\left(73^{\circ}\right.$ Deg.) this parameter can exceed 400 $\mathrm{nm} / \mathrm{RIU}$. Note that such a difference of sensitivities was recorded for a largely dominating majority of samples of single and double nanodot arrays, independently of the geometry of the unit cell (Table 1). Here, the samples typically demonstrate sensitivity in the range of 260-290 nm/RIU below the TIR angle and improved sensitivity of 370-410 nm/RIU above the TIR angle. On the other hand, as follows from Fig. 1d, substrate-related mode ( $\mathrm{S}+$ ) appears to be weakly sensitive to variations of RI (16-56 nm/RIU). In general, sensitivity of medium modes PSLR wat $_{\text {for }}$ different samples strongly 
correlates with the period of structures $(320 \mathrm{~nm})$, similarly to other transducers based on diffraction phenomena such as it was observed for surface plasmon polaritons over nanohole thin film arrays ${ }^{28}$.

It is important to consider the sharpness of measured resonances and thus examine system efficiency to measure small wavelength changes. For that purpose, one normally uses a characteristic "Figure of Merit" (FOM) parameter ${ }^{29}$ : $\mathrm{FOM}=(\Delta \lambda / \Delta n)(1 / \Delta \omega)$, where $\Delta \omega$ is the width of resonance at FWHM and $\Delta \lambda$ is the resonance shift for a $\Delta n$ refractiveindex change. The essence of FOM is straightforward: it adequately quantifies the sensing potential of plasmonic modes in configurations similar to those used in commercial instruments. Typical FOMs do not exceed 8 and 23 for the sensors based on $\mathrm{LPR}^{13,14}$ and $\mathrm{SPR}^{3}$, respectively. The employment of PSLRs enables one to increase these parameters up to 10 , 100 and more. In particular, in our experiments FOM reached $\sim 180$, which is in a good agreement with our earlier works where it reached $\sim 200-250^{22}$. As another illustration, it was shown ${ }^{30}$ that PSLRs in ordered metamaterial arrays of gold nanoparticles can offer a sensing performance that is at least an order of magnitude higher than uncoupled LPRs associated with disordered particle arrays. Other studies experimentally demonstrated a high-performance RI-sensitive sensor based on PSLR, while its FOM reached 38 normal incidence ${ }^{31}$.

Even when sensitivities of PSLR modes can be obtained within diffraction theory ${ }^{32}$, a detailed consideration of the phenomenon should take into account not only the dependence of diffraction edge position on RI of the environment, but also accompanying effects such as a certain mismatch of actual PSLR positions with that of the Rayleigh cut-off wavelengths and evanescent near-fields in the case of ATR geometry. However, in the first approximation, this sensitivity

Table 1. Summary of results, obtained for different PSLR modes from series of double dot samples in ATR geometry and corresponding sensitivity.

\begin{tabular}{|c|c|c|c|c|c|}
\hline $\begin{array}{l}\text { Distance } \\
\text { between } \\
\text { nanoparticles }\end{array}$ & Geometry & $\begin{array}{l}\text { Related } \\
\text { RA }\end{array}$ & $\begin{array}{l}\text { Angle } \\
\theta, \text { deg }\end{array}$ & $\begin{array}{l}\text { Experimental } \\
\text { sensitivity, } \\
\text { nm/RIU }\end{array}$ & $\begin{array}{l}\text { Analytical } \\
\text { sensitivity, } \\
\text { nm/RIU }\end{array}$ \\
\hline $190 \mathrm{~nm}$ & ATR & $\mathrm{S}+$ & 67 & 18 & 0 \\
\hline $195 \mathrm{~nm}$ & ATR (before TIR) & $\mathrm{W}+$ & 47 & 305 & 320 \\
\hline $195 \mathrm{~nm}$ & ATR & $\mathrm{W}+$ & 73 & 365 & 320 \\
\hline $134 \mathrm{~nm}$ & ATR (before TIR) & $\mathrm{S}+$ & 46.5 & 16 & 0 \\
\hline $134 \mathrm{~nm}$ & ATR & $\mathrm{S}+$ & 70 & 56 & 0 \\
\hline $134 \mathrm{~nm}$ & ATR (before TIR) & $\mathrm{W}+$ & 46.5 & 263 & 320 \\
\hline $134 \mathrm{~nm}$ & ATR & $\mathrm{W}+$ & 70 & 400 & 320 \\
\hline $170 \mathrm{~nm}$ & ATR (before TIR) & S+ & 46 & 11 & 0 \\
\hline $170 \mathrm{~nm}$ & ATR (before TIR) & $\mathrm{W}+$ & 46 & 291 & 320 \\
\hline $170 \mathrm{~nm}$ & ATR & $\mathrm{W}+$ & $\sim 70$ & 360 & 320 \\
\hline $142 \mathrm{~nm}$ & ATR & $\mathrm{S}+$ & 70 & 19 & 0 \\
\hline $142 \mathrm{~nm}$ & ATR & $\mathrm{W}+$ & 70 & 365 & 320 \\
\hline $128 \mathrm{~nm}$ & ATR & $\mathrm{S}+$ & 74 & 21 & 0 \\
\hline $128 \mathrm{~nm}$ & ATR & $\mathrm{W}+$ & 74 & 368 & 320 \\
\hline
\end{tabular}


should correlate with the sensitivity of the diffraction edge. The position of this Rayleigh cut-off wavelength for water and substrate modes in ATR geometry can be derived as follows:

$$
\begin{aligned}
& \pm \lambda_{R_{-} A T R}^{\text {Wat }}=a\left(n_{\text {sub }} \sin \theta \pm n_{\text {Wat }}\right) \\
& \pm \lambda_{R_{-} A T R}^{\text {Sub }}=a n_{\text {sub }}(\sin \theta \pm 1)
\end{aligned}
$$

Therefore, these sensitivities can be expressed as follows:

$$
\begin{aligned}
& S_{A T R}\left(P S L R_{w a t}\right)=\frac{\partial \lambda_{R_{-} A T R}^{w a t}}{\partial n}=\frac{\partial}{\partial n}\left[a\left(n_{\text {sub }} \sin \theta \pm n\right)\right]= \pm a \\
& S_{A T R}\left(P S L R_{\text {sub }}\right)=\frac{\partial \lambda_{R_{A} A T R}^{S u b}}{\partial n}=\frac{\partial}{\partial n}\left[a n_{\text {sub }}(\sin \theta \pm 1)\right]=0
\end{aligned}
$$

Here, the sensitivity of the medium mode (PSLR wat) appears to be linked to the period of the structure ( $a \mathrm{~nm} / \mathrm{RIU})$, while the substrate mode should be insensitive to these variations.

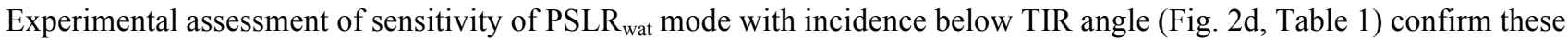
theoretical predictions, however, a higher sensitivity under angles exceeding the TIR angle is a pleasant surprise taking into account the fact that this sensitivity does not originate from diffraction theory considerations. Results show that this effect is probably related to the involvement of evanescent wave under TIR, which can additionally react on RI variations.

\section{CONCLUSION}

In this paper, conditions of excitation and properties of diffractively coupled plasmonic surface lattice resonances over 2D metamaterial array of double nanoparticles in ATR geometry were established. Generation of PSLR $\mathrm{a}_{\mathrm{air}} / \mathrm{PSLR}_{\mathrm{wat}}$ and PSLR $_{\text {sub }}$ plasmonic modes, corresponding to the coupling of individual plasmon oscillations at medium- and substraterelated diffraction cut-off edges was identified. Their sensitivities to the variations of refractive index of adjacent sample dielectric medium were assessed. The determined spectral sensitivity of medium-related PSLRs $(300-400 \mathrm{~nm} / \mathrm{RIU})$ is conditioned by nanostructure periodicity, while substrate-related modes showed much lower intensity. As follows from a good coincidence between the proposed theory and the experimental data, this sensitivity level is mainly conditioned by a Rayleigh cut-off spectral shift due to medium RI variation. However, near-field effects and other conditions must be taken into account to describe unexpected rise of sensitivity after TIR angle. Combining advantages of nanoscale architectures, including drastic concentration of electric field, possibility for manipulations at the nanoscale and high phase and spectral sensitivities, PSLR $_{\mathrm{s}}$ promise the advancement of current state-of-the-art plasmonic biosensing technology toward single molecule label-free detection.

\section{ACKNOWLEDGEMENTS}

AD, GT, AVK acknowledge the support from A*MIDEX project (No. ANR-11-IDEX-0001-02) funded by the "Investissements d'Avenir" French Government program, managed by the ANR, and LASERNANOCANCER and GRAVITY projects of ITMO "Plan Cancer 2014-2019" INSERM program. ANG acknowledges the support from the European Union's Horizon 2020 research and innovation program under grant agreement No. 696656 "GrapheneCore1", the EPSRC (EP/K011022/1) and SONY research agreement.

\section{REFERENCES}

[1] Liedberg, B., Nylander, C. and Lundström, I., "Biosensing with surface plasmon resonance - how it all started," Biosens. Bioelectron. 10(8) (1995).

[2] Liedberg, B., Nylander, C. and Lunström, I., "Surface plasmon resonance for gas detection and biosensing," Sensors and Actuators 4(C), 299-304 (1983). 
[3] Zhang, L.-M. and Uttamchandani, D., "Optical chemical sensing employing surface plasmon resonance," Electron. Lett. 24(23), 1469 (1988).

[4] Kabashin, A. V and Nikitin, P. I., "Interferometer based on a surface-plasmon resonance for sensor applications," Quantum Electron. 27(7), 653-654 (1997).

[5] Kabashin, A. V., Kochergin, V. E., Beloglazov, A. A. and Nikitin, P. I., "Phase-polarisation contrast for surface plasmon resonance biosensors," Biosens. Bioelectron. 13(12), 1263-1269 (1998).

[6] Kabashin, A. V., Kochergin, V. E. and Nikitin, P. I., "Surface plasmon resonance bio- and chemical sensors with phase-polarisation contrast," Sensors Actuators, B Chem. 54(1), 51-56 (1999).

[7] Homola, J., [Surface Plasmon Resonance Based Sensors] (2006).

[8] Tudos, A. J. and Schasfoort, R. B. M., "Handbook of Surface Plasmon Resonance" (2008).

[9] Patskovsky, S., Kabashin, A. V, Meunier, M. and Luong, J. H. T., "Silicon-based surface plasmon resonance sensing with two surface plasmon polariton modes.," Appl. Opt. 42(34), 6905-6909 (2003).

[10] Piliarik, M., Homola, J., Maníková, Z. and Čtyroký, J., "Surface plasmon resonance sensor based on a singlemode polarization-maintaining optical fiber," Sensors Actuators, B Chem. 90(1-3), 236-242 (2003).

[11] Nemova, G., Kabashin, A. V. and Kashyap, R., "Surface plasmon-polariton Mach-Zehnder refractive index sensor," J. Opt. Soc. Am. B 25(10), 1673 (2008).

[12] Hassani, A., Gauvreau, B., Fehri, M. F., Kabashin, A. and Skorobogatiy, M., "Photonic crystal fiber and waveguide-based surface plasmon resonance sensors for application in the visible and near-IR," Electromagnetics 28(3), 198-213 (2008).

[13] Haes, A. J. and Van Duyne, R. P., "A nanoscale optical biosensor: sensitivity and selectivity of an approach based on the localized surface plasmon resonance spectroscopy of triangular silver nanoparticles.," J. Am. Chem. Soc. 124(35), 10596-10604 (2002).

[14] Anker, J. N., Hall, W. P., Lyandres, O., Shah, N. C., Zhao, J. and Van Duyne, R. P., "Biosensing with plasmonic nanosensors.," Nat. Mater. 7(6), 442-453 (2008).

[15] Kabashin, A. V, Evans, P., Pastkovsky, S., Hendren, W., Wurtz, G. A., Atkinson, R., Pollard, R., Podolskiy, V. A. and Zayats, A. V., "Plasmonic nanorod metamaterials for biosensing," Nat. Mater. 8(11), 867-871 (2009).

[16] Sreekanth, K. V., Alapan, Y., ElKabbash, M., Ilker, E., Hinczewski, M., Gurkan, U. A., De Luca, A. and Strangi, G., "Extreme sensitivity biosensing platform based on hyperbolic metamaterials," Nat. Mater. 15(6), 621-627 (2016).

[17] Aristov, A. I., Manousidaki, M., Danilov, A., Terzaki, K., Fotakis, C., Farsari, M., Kabashin, A. V. 3D plasmonic crystal metamaterials for ultra-sensitive biosensing. Sci. Rep., 6, 25380 (2016).

[18] Kravets, V. G., Schedin, F. and Grigorenko, A. N., "Extremely narrow plasmon resonances based on diffraction coupling of localized plasmons in arrays of metallic nanoparticles," Phys. Rev. Lett. 101(8), 1-4 (2008).

[19] Auguié, B. and Barnes, W. L., "Collective Resonances in Gold Nanoparticle Arrays," Phys. Rev. Lett. 101(14), 143902 (2008).

[20] Chu, Y., Schonbrun, E., Yang, T. and Crozier, K. B., "Experimental observation of narrow surface plasmon resonances in gold nanoparticle arrays," Appl. Phys. Lett. 93(18), 2006-2009 (2008).

[21] Kravets, V. G., Schedin, F., Jalil, R., Britnell, L., Gorbachev, R. V, Ansell, D., Thackray, B., Novoselov, K. S., Geim, a K., Kabashin, a V and Grigorenko, A. N., "Singular phase nano-optics in plasmonic metamaterials for label-free single-molecule detection," Nat. Mater. 12(4), 304-309 (2013).

[22] Kravets, V. G., Schedin, F., Kabashin, A. V and Grigorenko, A. N., "Sensitivity of collective plasmon modes of gold nanoresonators to local environment.," Opt. Lett. 35(7), 956-958 (2010).

[23] Kabashin, A. V., Patskovsky, S., Grigorenko, A. N., "Phase and amplitude sensitivities in Surface Plasmon Resonance bio- and chemical sensing. Opt. Express," 17, 21191-21204 (2009).

[24] Law, W.-C., Markowicz, P., Yong, K.-T., Roy, I., Baev, A., Patskovsky, S., Kabashin, A. V., Ho, H.-P. and Prasad, P. N., "Wide dynamic range phase-sensitive surface plasmon resonance biosensor based on measuring the modulation harmonics," Biosens. Bioelectron. 23(5), 627-632 (2007).

[25] Huang, Y. H., Ho, H. P., Kong, S. K. and Kabashin, A. V., "Phase-sensitive surface plasmon resonance biosensors: Methodology, instrumentation and applications," Ann. Phys. 524(11), 637-662 (2012).

[26] Danilov, A., Tselikov, G., Wu, F., Kravets, V. G., Ozerov, I., Bedu, F., Grigorenko, A. N. and Kabashin, A. V., "Ultra-narrow surface lattice resonances in plasmonic metamaterial arrays for biosensing applications," Biosens. Bioelectron. 104, 102-112 (2018).

[27] Nikitin, A. G., Kabashin, A. V and Dallaporta, H., "Plasmonic resonances in diffractive arrays of gold nanoantennas: Near and far field effects," Opt. Express 20(25), 27941-27952 (2012). 
[28] Brolo, A. G., Gordon, R., Leathem, B. and Kavanagh, K. L., "Surface plasmon sensor based on the enhanced light transmission through arrays of nanoholes in gold films.," Langmuir 20(17), 4813-4815 (2004).

[29] Mayer, K. M., Hafner, J. H. and Antigen, A. À., "Localized surface plasmon resonance sensors," Chem. Rev. 111(6), 3828-3857 (2011).

[30] Offermans, P., Schaafsma, M. C., Rodriguez, S. R. K., Zhang, Y., Crego-Calama, M., Brongersma, S. H. and Gomez Rivas, J., "Universal scaling of the figure of merit of plasmonic sensors," ACS Nano 5(6), 5151-5157 (2011).

[31] Li, Y., An, B., Jiang, S., Gao, J., Chen, Y. and Pan, S., "Plasmonic induced triple-band absorber for sensor application," Opt. Express 23(13), 17607 (2015).

[32] Born, M., Wolf, E [Principles of Optics], Cambridge University Press, Cambridge (1999). 